\title{
Microstructural Developments Leading to New Advanced High Strength Sheet Steels: A Historical Assessment of Critical Metallographic Observations
}

\author{
David K. Matlock ${ }^{1}$, Larrin S. Thomas ${ }^{1}$, Mark D. Taylor ${ }^{1}$, Emmanuel De Moor ${ }^{1}$, and John G. Speer ${ }^{1}$ \\ 1. Advanced Steel Processing and Products Research Center, The George S. Ansell Department of \\ Metallurgical and Materials Engineering, Colorado School of Mines, Golden, Colorado 80401
}

Critical needs for new high strength steels to enable design of lighter weight systems which incorporate components that operate at higher stress levels than previously specified have led to the development of multiple new steel grades in all product forms. Of particular interest here are the extensive recent developments that have been employed to produce and implement new advanced high strength sheet steel (AHSS) grades in affordable lighter-weight vehicles with significantly enhanced fuel efficiency and safety [1]. Multiple different alloying and processing strategies have been considered [1], and designations for different AHSS grades, characterized by specific tensile strength-ductility combinations, have evolved. "First generation” AHSS grades include DP (dual-phase), CP (complex phase) and TRIP (transformation induced plasticity) steels. Current emphasis is on enhancing properties of first generation AHSS [2] while developing new "third generation” AHSS grades including Q\&P steels (quenched and partitioned), TBF (TRIP aided bainitic ferrite) steels, and others with the higher strength-ductility combinations required by vehicle designers [1].

Initially DP steels were envisioned to contain a two-phase microstructure of ferrite and martensite produced by quenching a low carbon steel from an intercritical annealing temperature. However, early research in the 1970's showed that DP steels contained complex combinations of other constituents including retained austenite and bainite. Many of the new AHSS grades have evolved from similar underlying principles of microstructural evolution in DP steels, and developments have relied on systematic microstructural analyses that have incorporated conventional light and electron microscopic techniques as well as many of the advanced techniques available today. In this paper microstructural observations from early research on DP steels are contrasted with current results on new AHSS grades to illustrate the importance that microstructural analyses have had to advance modern steels.

Early DP steel research showed that the resulting microstructures were complex, sensitive to both alloy content and time-temperature processing history, and contained other constituents, including retained austenite, that contributed to the observed unique mechanical properties. Specialized etching techniques based on multi-step and color etching were employed to highlight specific microstructural constituents as illustrated in Figure 1 [3]. Figure 1 reveals that the final ferrite present at room temperature is comprised of intercritical ferrite (i.e. present at the annealing temperature) and epitaxial ferrite formed on cooling as carbon redistributed to martensite and other high-carbon transformation products. Modifications to classic DP alloys and thermal process histories now incorporate alternate cooling cycles leading to the development of TRIP, TBF, and Q\&P steels, with microstructures designed to contain increased amounts of retained austenite, a critical constituent in newer AHSS grades [1]. Multiple conventional and advanced metallographic techniques have been employed to evaluate the refined microstructures, often containing sub-micron sized constituents. Determination of the amount, morphology, and distribution of retained austenite is one important outcome [4]. For a Q\&P steel, Figures $2 \mathrm{a}$ and $2 \mathrm{~b}$ compare secondary electron and EBSD images and show the distribution of retained austenite, information essential to assessing, and potentially modifying, imposed thermal cycles [5]. 
Figures 2c and 2d illustrate, in a study on hydrogen embrittlement in TRIP steels, how application of a modified hydrogen microprint technique shows clear evidence of hydrogen in ferrite but essentially absent in the austenite containing constituent. This latter point is important for AHSS grades that may be exposed to hydrogen.

AHSS advances continue, in part as a direct result of the application of modern analytical tools that are becoming routinely available and used to comprehensively assess the ultra-fine non-equilibrium microstructures currently being produced, often with important composition gradients within individual constituents. Recently applications involving atom probe tomography, nano secondary ion mass spectroscopy, and Mössbauer spectroscopy [6] have provided valuable information to interpret the fine features associated with new AHSS products, and research combining all technologies leading to new steel products will continue [7].

[1] E. De Moor et al., AIST Trans., Iron and Steel Technology, 7, no. 11 (2010), p. 133.

[2] M. D. Taylor et al., J. of Mater. Engr. and Performance, 23 (2014), p. 3685.

[3] R.D. Lawson et al., Metallography, 13 (1980), p. 71.

[4] G.A. Thomas et al., Microscopy and Microanalysis, 17 (2011), p. 368.

[5] J.A. Ronevich et al., Metallography, Microstructure, and Analysis, 1 (2012), p. 79.

[6] D.T. Pierce et al., Acta Materialia, in press, (2015) DOI: 10.1016/j.actamat.2015.01.024.

[7] The authors acknowledge the support of the corporate sponsors of the Advanced Steel Processing and Products Research Center at the Colorado School of Mines.
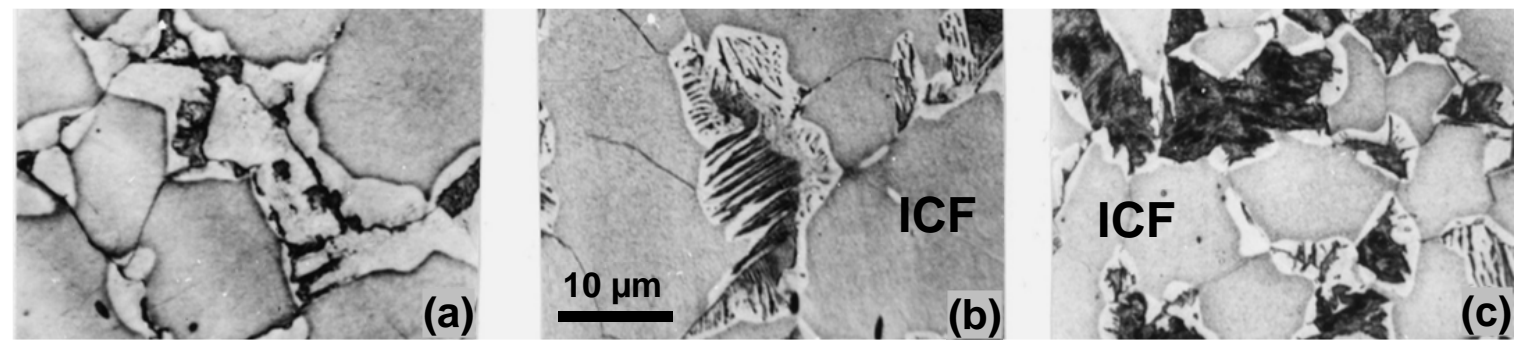

Figure 1. Intercritical ferrite (ICF) (grey) and epitaxial ferrite (white) revealed after cooling a 0.08C1.47Mn-0.34Si-0.053Nb from a $4 \mathrm{~min}$ anneal at $810{ }^{\circ} \mathrm{C}, 12{ }^{\circ} \mathrm{C} / \mathrm{s}$ (a), $135{ }^{\circ} \mathrm{C} / \mathrm{s}$ (b), and $1000{ }^{\circ} \mathrm{C} / \mathrm{s}$ (c); (etched with picral followed by alkaline chromate staining) [3].
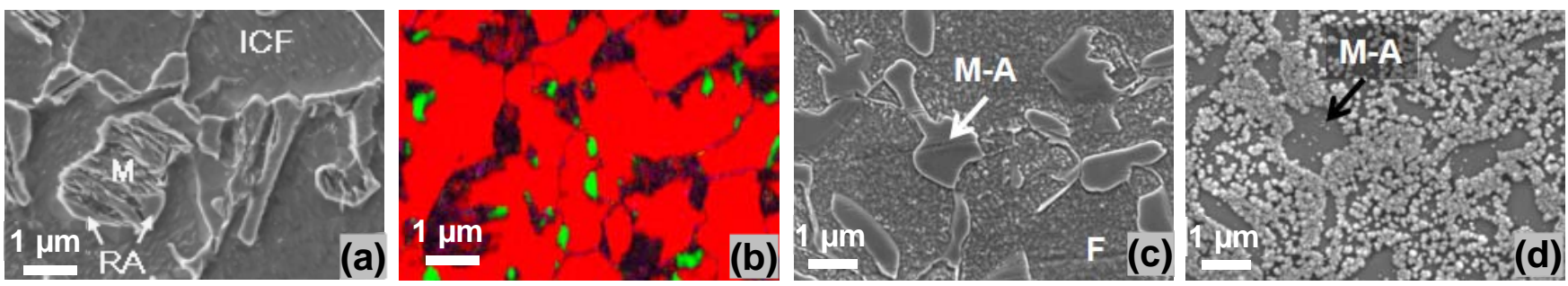

Figure 2. Example images of retained austenite in 0.19C-1.59Mn-1.63Si steel with different AHSS processing histories: Q\&P steel where (a) and (b) are secondary electron and EBSD images (different areas of same sample), respectively. In (b) austenite = green, ferrite and martensite = red, carbide structures and martensite with reduced image quality appear dark [4]. ((a) and (c) 2 pct nital etch); TRIP steel where (c) is SEM image of as processed steel and (d) after hydrogen charging and preparation with the modified hydrogen microprint technique showing hydrogen concentrated in the ferrite constituent [5]. 